Research Article

\title{
Lie Symmetry Analysis and Explicit Solutions for the Time-Fractional Regularized Long-Wave Equation
}

\author{
Nisrine Maarouf (D, Hicham Maadan (D, and Khalid Hilal $(\mathbb{D}$ \\ Department of Mathematics, Sultan Moulay Slimane University, BP 523, 23000 Beni Mellal, Morocco \\ Correspondence should be addressed to Nisrine Maarouf; nisrine.maarouf6@gmail.com
}

Received 28 October 2020; Revised 15 December 2020; Accepted 10 January 2021; Published 2 February 2021

Academic Editor: Ram n Quintanilla

Copyright (c) 2021 Nisrine Maarouf et al. This is an open access article distributed under the Creative Commons Attribution License, which permits unrestricted use, distribution, and reproduction in any medium, provided the original work is properly cited.

\begin{abstract}
This paper systematically investigates the Lie group analysis method of the time-fractional regularized long-wave (RLW) equation with Riemann-Liouville fractional derivative. The vector fields and similarity reductions of the time-fractional (RLW) equation are obtained. It is shown that the governing equation can be transformed into a fractional ordinary differential equation with a new independent variable, where the fractional derivatives are in Erdèlyi-Kober sense. Furthermore, the explicit analytic solutions of the time-fractional (RLW) equation are obtained using the power series expansion method. Finally, some graphical features were presented to give a visual interpretation of the solutions.
\end{abstract}

\section{Introduction}

In recent decades, fractional differential equations have been used to describe several natural phenomena in applied sciences such as fluid flow, chemistry, physics, biology, and other areas [1-5], for modeling different phenomena which may depend on the previous time as well as the current time. Besides, fractional differential equations can be considered more efficient to investigate the process of scientific phenomena with complex irregular conditions, see, for example, [6-11]. Therefore, a large number of powerful methods have been developed to look for exact and numerical solutions such us the variational iteration method [12], transformation method [13], finite-difference method [14], Exp-function method [15], homotopy analysis method [16], Adomian decomposition method [17], the first integral method [18], and sine-cosine method [19].

Lie symmetry analysis was advocated by the Norwegian mathematician Sophis Lie (1842-1899) in the beginning of the nineteenth century, which is one of the most powerful methods for studying nonlinear partial differential equations and looking for its analytical solutions. It has several applications including linearization of some nonlinear equations, construction of new solutions from trivial ones, construction of integrator factor, reduction of order, and reduction of the independent variables.
Gazizov et al. [20,21] are the first who started rigorous studies of symmetries admitted by fractional differential equations focusing on Riemann-Liouville and Caputo derivatives. In [22], Wang and Xu investigated the symmetry properties of the timefractional KDV equation (see also [23]). It is worth to mention that Yusuf [24] analyzed the equation for fluid flow in porous media using the Lie symmetry method and invariant subspace method for constructing exact solutions and conservation laws of the equation. In addition, the authors of [25] have made an attempt to apply the Lie group method to the time-fractional generalized Burgers and Korteweg de Vries equations. In [26], authors have investigated the Lie symmetry analysis of timefractional Harry-Dym equation with Riemann-Liouville derivative to obtain invariant solutions and symmetry reductions. Moreover, the interested reader can be also referred to the following papers and books [22, 25, 27-32].

In this paper, we investigate the time-fractional regularized long-wave equation given by

$$
D_{t}^{\alpha} u+u_{x}-\frac{a}{2} u u_{x}-b u_{x x t}=0,
$$

where $D_{t}^{\alpha}$ is the fractional derivative of order $0<\alpha \leq 1$ and $a$ and $b$ are arbitrary constants. 
The regularized long-wave (RLW) equation was first introduced by Peregrine [33] to describe the development of an undular bore. It is one of the most important nonlinear evolution equations which have been used to model many physical phenomena such as shallow water waves and ionacoustic plasma waves. Many researchers have tried in the past to construct solution of the nonlinear regularized long- wave (RLW) equation [34-36]. Especially, fractional version of this model has been studied (see $[37,38]$ ).

We would like to mention that there is no unique definition of fractional derivatives $[5,11]$; in this paper, we use the Riemann-Liouville fractional derivative of order $\alpha$ $(\alpha>0)$ which is defined by

$$
D^{\alpha} u(t, x)=\frac{\partial^{\alpha} u}{\partial t^{\alpha}}=\left\{\begin{array}{l}
\frac{\partial^{m} u}{\partial t^{m}}, \alpha=m \in \mathbb{N} \\
\frac{1}{\Gamma(m-\alpha)} \frac{\partial^{m}}{\partial t^{m}} \int_{0}^{t}(t-\tau)^{m-\alpha-1} u(\tau, x) \mathrm{d} \tau, m-1<\alpha<m, m \in \mathbb{N},
\end{array}\right.
$$

where $\Gamma(\mathrm{z})$ is the Gamma function defined by

$$
\Gamma(z)=\int_{0}^{+\infty} e^{-t} t^{z-1}
$$

The main purpose of this paper is to investigate the symmetry approach for determining the Lie point symmetries and symmetry reductions of the time-fractional regularized long-wave equation (RLW). Then, we have shown that the time fractional (RLW) can be transformed into an ordinary differential equation of fractional order with Erdèlyi-Kober fractional differential derivative. Using the power series method [39], the exact solutions of the time-fractional (RLW) equation are derived.

The remainder of this paper is organized as follows: in Section 2, the general procedure of the Lie symmetry method for fractional partial differential equations (FPDEs) is presented. By employing the proposed method, Lie point symmetries of equation (1) are obtained in Section 3. In Section 4, by using similarity variables, the reduced equations are obtained, solving some of them, and then the similarity solutions of equation (1) are deduced. Section 5 is devoted to constructing the explicit analytical power series solutions. Some graphical features for the obtained solutions are presented in Section 6. Finally, a brief conclusion is given in Section 7.

\section{The General Procedure of Lie Symmetry Analysis Method for FNPDE}

In this section, we present brief details of Lie symmetry analysis for fractional partial differential equations (FPDEs) of the form

$$
D_{t}^{\alpha} u=F\left(x, t, u, u_{t}, u_{x}, u_{x t}, u_{x x}, u_{x x t}, \ldots\right), \quad \alpha>0
$$

We assume that equation (4) is invariant under a one parameter $\varepsilon$ continuous transformations and the construction of the symmetry group is equivalent to the determination of its infinitesimal transformations:

$$
\begin{aligned}
\widehat{x} & =x+\varepsilon \xi(x, t, u)+0\left(\varepsilon^{2}\right), \\
\widehat{t} & =t+\varepsilon \tau(x, t, u)+0\left(\varepsilon^{2}\right), \\
\widehat{u} & =u+\varepsilon \eta(x, t, u)+0\left(\varepsilon^{2}\right), \\
D_{t}^{\alpha} \widehat{u} & =D_{t}^{\alpha} u+\varepsilon \eta_{\alpha}^{0}(x, t, u)+0\left(\varepsilon^{2}\right), \\
\frac{\partial^{3} \widehat{u}}{\partial \widehat{x}} & =\frac{\partial u}{\partial x}+\varepsilon \eta^{x}(x, t, u)+0\left(\varepsilon^{2}\right), \\
\frac{\partial^{3} \widehat{u}}{\partial^{2} \widehat{x} \partial \widehat{t}} & =\frac{\partial u}{\partial^{2} x \partial t}+\varepsilon \eta^{x x t}(x, t, u)+0\left(\varepsilon^{2}\right), \\
\vdots &
\end{aligned}
$$

where $\varepsilon<<1$ is a group parameter and $\xi, \eta$, and $\tau$ are the infinitesimals of transformations for the dependent and independent variables, respectively. The explicit expressions of $\eta x$ and $\eta x x t$ are given by

$$
\begin{aligned}
\eta^{x} & =D_{x}(\eta)-u_{x} D_{x}(\xi)-u_{t} D_{x}(\tau), \\
\eta^{t} & =D_{t}(\eta)-u_{x} D_{t}(\xi)-u_{t} D_{t}(\tau), \\
\eta^{x t} & =D_{x}(\eta t)-u_{x t} D_{x}(\xi)-u_{t t} D_{x}(\tau), \\
\eta^{x x t} & =D_{x}\left(\eta_{x t}\right)-u_{x x x} D_{x}(\xi)-u_{x x t} D_{x}(\tau), \\
\vdots &
\end{aligned}
$$

where $D_{x}$ and $D_{t}$ are the total derivatives with respect to $x$ and $t$, respectively, which are defined as

$$
D_{x^{i}}=\frac{\partial}{\partial x^{i}}+u_{i} \frac{\partial}{\partial u}+u_{i j} \frac{\partial}{\partial u_{j}}+\ldots, \quad i, j=1,2,3, \ldots,
$$

where $u_{i}=\partial u /\left(\partial x^{i}\right), u_{i j}=\partial^{2} u / \partial x_{i} \partial x_{j}$, and so on.

The infinitesimal generator $X$ is represented by the following expression:

$$
X=\xi(x, t, u) \frac{\partial}{\partial x}+\tau(x, t, u) \frac{\partial}{\partial t}+\eta(x, t, u) \frac{\partial}{\partial u},
$$

Since the lower limit of integral (2) is fixed, so the structure of the Riemann-Liouville derivative must be 
invariant under transformations (5). The invariance condition yields

$$
\left.\tau(x, t, u)\right|_{t=0}=0 .
$$

The $\alpha$-th extended infinitesimal related to the Riemann-Liouville fractional time derivative (see $[20,21]$ ) can be written as follows:

$$
\begin{aligned}
\eta_{\alpha}^{0} & =D_{t}^{\alpha}(\eta)+\xi D_{t}^{\alpha}\left(u_{x}\right)-D_{t}^{\alpha}\left(\xi u_{x}\right) \\
& +D_{t}^{\alpha}\left(D_{t}(\tau) u\right)-D_{t}^{\alpha+1}(\tau u)+\tau D_{t}^{\alpha+1}(u),
\end{aligned}
$$

where the total fractional derivative operator is denoted as $D_{t}^{\alpha}$.

Here, for making equation (10) more general, the generalized Leibnitz rule [5] has been presented, which is given as

$$
D_{t}^{\alpha}\left((f(t) g(t))=\sum_{n=1}^{\infty}\left(\begin{array}{l}
\alpha \\
n
\end{array}\right) D_{t}^{\alpha-n}(f(t)) D_{t}^{n} g(t), \quad \alpha>0,\right.
$$

where

$$
\left(\begin{array}{l}
\alpha \\
n
\end{array}\right)=\frac{\Gamma(n-\alpha)(-1)^{n-1} \alpha}{\Gamma(1-\alpha) \Gamma(n+1)}
$$

Now, by using Leibnitz rule as presented below, we get

$$
\begin{aligned}
\eta_{\alpha}^{0}= & D_{t}^{\alpha}(\eta)-\alpha D_{t}(\tau) D_{t}^{\alpha} u-\sum_{n=1}^{\infty}\left(\begin{array}{l}
\alpha \\
n
\end{array}\right) D_{t}^{n}(\xi) D_{t}^{\alpha-n} u_{x} \\
& -\sum_{n=1}^{\infty}\left(\begin{array}{c}
\alpha \\
n+1
\end{array}\right) D_{t}^{n}(\tau) D_{t}^{\alpha-n}(u) .
\end{aligned}
$$

Then, the chain rule for composite function [5] can be written as

$$
\frac{\mathrm{d}^{n} \phi(h(t))}{\mathrm{d} t^{n}}=\sum_{k=0}^{n} \sum_{r=0}^{k}\left(\begin{array}{l}
\alpha \\
n
\end{array}\right) \frac{1}{k !}[-h(t)]^{r} \frac{\mathrm{d}^{n}}{\mathrm{~d} t^{n}}\left[(h(t))^{k-r}\right] \frac{\mathrm{d}^{k} \phi(h)}{\mathrm{d} h^{k}} .
$$

By applying this rule and the generalized Leibnitz rule with $f(t)=1$, we have

$$
D_{t}^{\alpha}(\eta)=\frac{\partial^{\alpha} \eta}{\partial t^{\alpha}}+\eta_{u} \frac{\partial^{\alpha} u}{\partial t^{\alpha}}-u \frac{\partial^{\alpha} \eta_{u}}{\partial t^{\alpha}}+\sum_{n=1}^{\infty}\left(\begin{array}{c}
\alpha \\
n
\end{array}\right) \frac{\partial^{\alpha} \eta_{u}}{\partial t^{n}} D_{t}^{\alpha-n}(u)+\mu,
$$

where

$$
\begin{gathered}
\mu=\sum_{n=2}^{\infty} \sum_{m=2}^{n} \sum_{k=2}^{m} \sum_{r=0}^{k-1}\left(\begin{array}{c}
\alpha \\
n
\end{array}\right)\left(\begin{array}{l}
n \\
m
\end{array}\right)\left(\begin{array}{l}
k \\
r
\end{array}\right) \frac{1}{k !} \frac{\left(t^{n-\alpha}\right)}{(\tau(n+1-\alpha))} \\
\cdot(-u)^{r} \frac{\partial^{m}}{\partial t^{m}}\left(u^{k-r}\right) \frac{\partial^{n-m+k} \eta}{\partial x^{n-m} \partial u^{k}}
\end{gathered}
$$

Therefore, the $\alpha$-th extended infinitesimal given in (13) becomes

$\eta_{\alpha}^{0}=\frac{\left(\partial^{\alpha} \eta\right)}{\left(\partial t^{\alpha}\right)}+\left(\eta_{u}-\alpha D_{t}(\tau)\right) \frac{\left(\partial^{\alpha} \eta u\right)}{\left(\partial t^{\alpha}\right)}-u \frac{\left(\partial^{\alpha} \eta_{u}\right)}{\left(\partial t^{\alpha}\right)}+\mu+\sum_{n=1}^{\infty}\left[\left(\begin{array}{c}\alpha \\ n\end{array}\right) \frac{\partial^{n} \eta_{u}}{\partial t^{n}}-\left(\begin{array}{c}\alpha \\ n+1\end{array}\right) D_{x}^{n+1}(\tau)\right] D_{t}^{\alpha-n}(u)-\sum_{n=1}^{\infty}\left(\begin{array}{c}\alpha \\ n\end{array}\right) D_{t}^{n}(\xi) D_{t}^{\alpha-n}\left(u_{x}\right)$

Theorem 1 (see [23])

A solution $u=\theta(x, t)$ is an invariant solution of equation

(4) if and only if

(i) $u=\theta(x, t)$ is an invariant surface; in other words,

$$
X \theta=0 \Leftrightarrow\left(\xi(x, t, u) \frac{\partial}{\partial x}+\zeta(x, t, u) \frac{\partial}{\partial t}+\eta(x, t, u) \frac{\partial}{\partial u}\right)
$$$$
\theta=0 .
$$

(ii) $u=\theta(x, t)$ is the solution of FPDE (4).

\section{Symmetry Analysis of Time-Fractional Regularized Long-Wave Equation}

We complete this section in the light of references $[20,21,25,29]$. We employ the Lie symmetry analysis to derive the similarity solution for nonlinear time-fractional equation (1) and to reduce it to be a FODE as shown in the next sections. 
Let us assume that equation (1) is invariant under oneparameter transformations (5), and we get the following transformed equation:

$$
D_{t}^{\alpha} \widehat{u}+\widehat{u}_{x}-\frac{a}{2} \widehat{u} \widehat{u}_{x}-b \widehat{u}_{x x t}=0 .
$$

Using point transformation equations (6) in equation (19), we obtain the following symmetry determining equation:

$$
\eta_{\alpha}^{0}-\left(1-\frac{a}{2} u\right) \eta^{x}-\frac{a}{2} \eta u_{x}-b \eta^{x x t}=0
$$

such that $u=u(x, t)$ satisfies equation (1).

By substituting the expressions $\eta_{\alpha}^{0}$ given in equations (6) and (17) into equation (20), we get

$$
\begin{aligned}
D_{t}^{\alpha} & (\eta)+\left(\eta_{u}-\alpha D_{t}(\tau)\right) D_{t}^{\alpha} u-u D_{t}^{\alpha} \eta_{u}+ \\
\mu & +\sum_{n=1}^{\infty}\left(\begin{array}{c}
\alpha \\
n
\end{array}\right) D_{t}^{n}(\xi) D_{t}^{\alpha-n} u_{x} \\
& +\sum_{n=1}^{\infty}\left[\left(\begin{array}{c}
\alpha \\
n
\end{array}\right) D_{t}^{n}\left(\eta_{u}\right)-\left(\begin{array}{c}
\alpha \\
n+1
\end{array}\right) D_{t}^{n+1}(\tau)\right] D_{t}^{\alpha-n}(u) \\
& +\left(1-\frac{a}{2} u\right)\left(\eta_{x}+u_{x}\left(\eta_{u}-\xi_{x}\right)-u_{x}^{2} \xi_{u}-u_{t} \tau_{x}-u_{t} u_{x} \tau_{u}\right)-\frac{a}{2} \eta u_{x} \\
& -b\left(\eta_{x x t}+u_{x}\left(2 \eta_{x t u}-\xi_{t x x}\right)+u_{x x}\left(\eta_{t u}-2 \xi_{t x}\right)+u_{x}^{2}\left(\eta_{t u u}-2 \xi_{t x u}\right)\right. \\
& +u_{t x x}\left(\eta_{u}-\tau_{t}-\xi_{x}-\tau_{x}\right)+u_{t x}\left(2 \eta_{u x}-2 \tau_{t x}-\xi_{x x}\right)+u_{x} u_{t x}\left(2 \eta_{u u}-4 \xi_{u x}-2 \tau_{t u}\right) \\
& +u_{t}\left(\eta_{u x x}-\tau_{t x x}\right)+u_{x} u_{t}\left(2 \eta_{u u x}-2 \tau_{t x u}-\xi_{u x x}\right)+u_{x x} u_{t}\left(\eta_{u u}-2 \xi_{u x}-\tau_{t u}\right) \\
& +u_{x}^{2} u_{t}\left(\eta_{u u u}-2 \xi_{u u x}-\tau_{t u u}\right)-3 u_{x} u_{x x} \xi_{t u}-u_{x}^{3} \xi_{t u u}-\xi_{u} u_{x x x} u_{t}-3 u_{x x} u_{t x} \xi_{u} \\
& -3 u_{x} u_{x x} u_{t} \xi_{u u}-u_{x} u_{t x x}\left(2 \xi_{u}+\tau_{u}\right)-3 u_{x}^{2} u_{t x} \xi_{u u u}-u_{x}^{3} u_{t} \xi_{u u u}-u_{t x}^{2} \tau_{u}-2 u_{t} u_{t x x} \tau_{u} \\
& -4 u_{t} u_{t x} \tau_{u x}-4 u_{x} u_{t} u_{t x} \tau_{u u}-u_{t}^{2} \tau_{u x x}-u_{x} u_{t}^{2}\left(2 \tau_{u u x}+\tau_{u u u}\right)-\tau_{u u} u_{x x} u_{t}^{2} \backslash \\
& -\tau_{x} u_{x t t}-2 \tau_{u x} u_{x} u_{t t}-u_{t t x} u_{x} \tau_{u}- \\
& \\
& \left.\left.+u_{x}^{2} u_{t t} \tau_{u u}-u_{x x x} u_{x} \xi_{u}+u_{t t} \tau_{x x}+u_{x} \xi_{u}\right)-u_{x x t}\left(\tau_{x}+u_{x} \tau_{u}\right)\right)=0, \\
& \left.+\xi_{x}\right)
\end{aligned}
$$


and equating various powers of derivatives of $u$ to zero, we obtain the following overdetermined system of linear equations

$$
\begin{aligned}
& \xi_{u}=\tau_{u}=\tau_{x} \\
& \xi_{u u}=\tau_{u u}=\tau_{x x}=\eta_{u u} \\
& \eta_{u t}-2 \xi_{x t}=0 \\
& 2 \xi_{x}+(1-\alpha) \tau_{t}=0, \\
& D_{t}^{n}(\xi)=0, \quad n=1,2,3, \ldots, \\
& \frac{\partial^{\alpha} \eta}{\partial t^{\alpha}}-u \frac{\partial^{\alpha} \eta_{u}}{\partial t^{\alpha}}+\left(1-\frac{a u}{2}\right) \eta_{x}-b \eta_{x x t}=0, \\
& \left(\begin{array}{c}
\alpha \\
n
\end{array}\right) \frac{\partial^{\alpha} \eta_{u}}{\partial t^{\alpha}}-\left(\begin{array}{c}
\alpha \\
n+1
\end{array}\right) D_{t}^{n+1}(\tau)=0, \quad n \in \mathbb{N} \\
& \left(\frac{a}{2} u-1\right) \xi_{x}-\frac{a}{2} \eta+\alpha\left(1-\frac{a u}{2}\right) \tau_{t}=0 .
\end{aligned}
$$

By solving the previous system, we get the following explicit form of infinitesimals:

$$
\begin{aligned}
\xi & =-\frac{C_{1} a(\alpha-1)}{\alpha+1} x+C_{3}, \\
\eta & =C_{1}(a u-2), \\
\tau & =-\frac{2 a C_{1}}{\alpha+1} t+C_{2},
\end{aligned}
$$

where $C_{1}, C_{2}$, and $C_{3}$ are arbitrary constants. Then, the Lie algebra of infinitesimal symmetries of equation (1) is given by

$$
\begin{aligned}
& X_{1}=\frac{\partial}{\partial x}, \\
& X_{2}=\frac{\partial}{\partial t}, \\
& X_{3}=-\frac{a(\alpha-1)}{\alpha+1} x \frac{\partial}{\partial x}+(a u-2) \frac{\partial}{\partial u}-\frac{2 a}{\alpha+1} t \frac{\partial}{\partial t} .
\end{aligned}
$$

Then, the infinitesimal generator of equation (1) can be written as follows:

$$
X=C_{1} X_{1}+C_{2} X_{2}+C_{3} X_{3} .
$$

\section{The Similarity Reduction of Fractional Regularized Long-Wave Equation}

In this section, we will use the characteristic equations of vector fields obtained in equations (24)-(26) for obtaining the reduction equations.

4.1. Case 1. The characteristic equation for infinitesimal generator (24) can be expressed symbolically as follows:

$$
\frac{\mathrm{d} x}{1}=\frac{\mathrm{d} t}{0}=\frac{\mathrm{d} u}{0} .
$$

By solving the above characteristic equation, we obtain the trivial solution.

4.2. Case 2. The characteristic equation for infinitesimal generator (25) can be expressed symbolically as follows:

$$
\frac{\mathrm{d} x}{0}=\frac{\mathrm{d} t}{1}=\frac{\mathrm{d} u}{0} \text {. }
$$

By solving the above characteristic equation, we obtain the trivial solution.

4.3. Case 3. The characteristic equation for infinitesimal generator (26) can be expressed as follows:

$$
\frac{\mathrm{d} x}{a((1-\alpha) /(\alpha+1)) x}=\frac{\mathrm{d} t}{((-2 a t) /(\alpha+1))}=\frac{\mathrm{d} u}{a u-2} .
$$

By solving the above characteristic equation, we obtain

$$
z=x t^{((1-\alpha) / 2)} \text {, }
$$

with the group invariant solution

$$
t^{((\alpha+1) / 2)}(a u-2)=f(z) .
$$

Hence, we get

$$
\begin{aligned}
u(t, x) & =\frac{1}{a} t^{(-(\alpha+1) / 2)} f(z)+\frac{2}{a}, \\
& =h(z)+\frac{2}{a},
\end{aligned}
$$

where $h(z)=(1 / a) t^{(-(\alpha+1) / 2)} f(z)$.

By means of this similarity transformation, time-fractional regularized long-wave equation (1) can be reduced to a nonlinear FODE with a new independent variable $z$. Thus, one can get the following theorem.

Theorem 2. Transformation (27) reduces equation (1) to the following nonlinear ordinary differential equation of fractional order:

$$
\left(P_{(2 / \alpha-1)}^{((1-3 \alpha / 2), \alpha)} f\right)(z)+2 \frac{t^{(\alpha+1 / 2)}}{\Gamma(1-\alpha)}-\frac{1}{2} f f_{z}+b \frac{(3 \alpha-1)}{2} f_{z z}=0,
$$

with the Erdelyi-Kober fractional operator [11],

$$
\begin{aligned}
\left(P_{\xi}^{\tau, \alpha} f\right)(z) & =\prod_{j=0}^{n-1}\left(\tau+j-\frac{1}{\xi} z \frac{\mathrm{d}}{\mathrm{d} z}\right)\left(K_{\xi}^{\tau+\alpha, n-\alpha} f\right)(z), \quad z>0, \xi>0, \alpha>0, \\
n & = \begin{cases}{[\alpha]+1,} & \alpha \notin \mathbb{N}, \\
\alpha, & \alpha \in \mathbb{N},\end{cases}
\end{aligned}
$$

where 


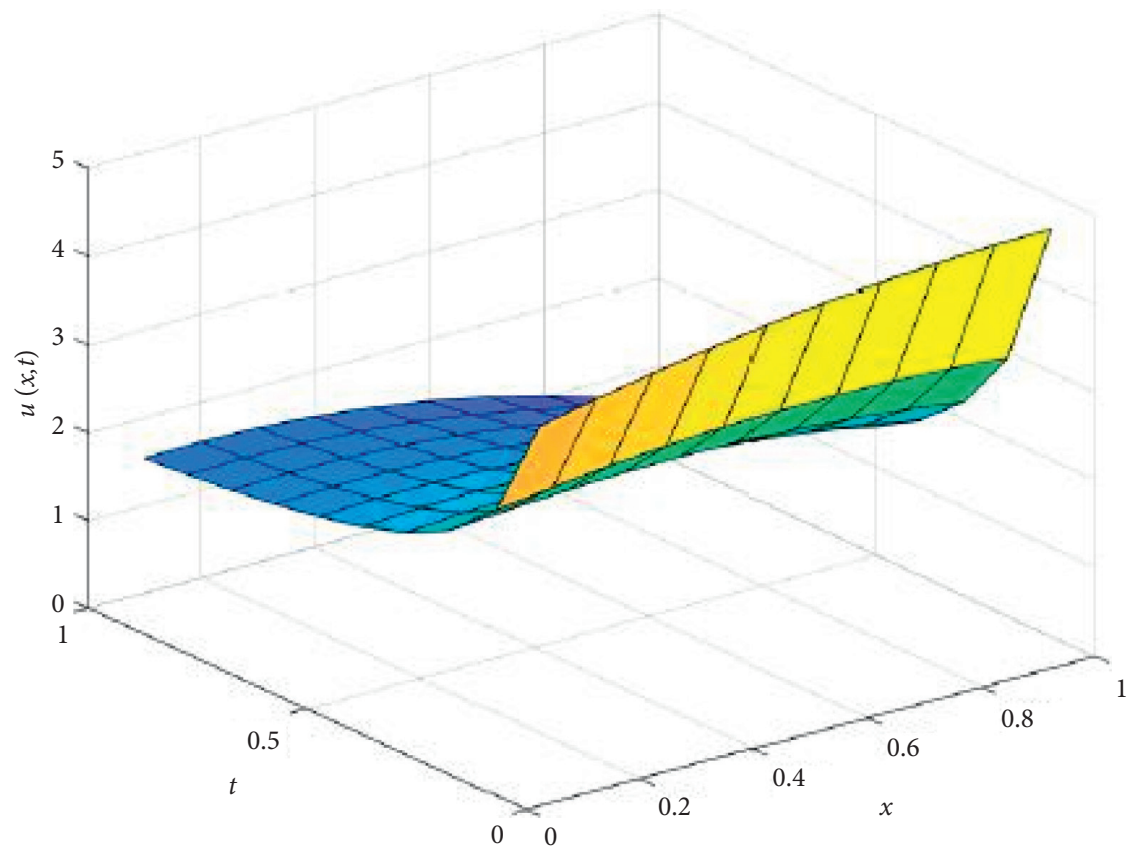

FIgURE 1: 3D plot of equation (52) with the parameter values $c_{0}=c_{1}=1, \alpha=0.5, a=2$, and $b=1$.

$$
\left(K_{\xi}^{\tau, \alpha} f\right)(z)= \begin{cases}\frac{1}{\Gamma(\alpha)} \int_{1}^{\infty}(v-1)^{\alpha-1} v^{-(\tau+\alpha)} f\left(z v^{(1 / \xi)}\right) \mathrm{d} v, & \alpha>0 \\ f(z), & \alpha=0\end{cases}
$$

which is the Erdelyi-Kober fractional integral operator.

Proof. Let $n-1<\alpha<n, n=1,2,3, \ldots$. The Riemann-Liouville fractional derivative becomes

$$
\begin{aligned}
D_{t}^{\alpha} h(z)= & \frac{1}{a} \frac{\partial^{n}}{\partial t^{n}}\left[\frac{1}{\Gamma(n-\alpha)} \int_{0}^{t}(t-s)^{n-\alpha-1} s^{(-(\alpha+1) / 2)}\right. \\
& \left.\cdot f\left(x s^{(1-\alpha / 2)}\right)\right] \mathrm{d} s .
\end{aligned}
$$

Let $\mathrm{d} s=-1 / s^{2} \mathrm{~d} v$. So, equation (38) can be written as

$$
\begin{aligned}
\frac{\partial^{n} h(z)}{\partial t^{n}}= & \frac{1}{a} \frac{\partial^{n}}{\partial t^{n}}\left[t^{n-((3 \alpha+1) / 2)} \frac{1}{\Gamma(n-\alpha)} \int_{1}^{\infty} v^{-(n-\alpha+(1-\alpha / 2))}\right. \\
& \left.\cdot(v-1)^{n-\alpha-1} f\left(z v^{(2 / 1-\alpha)}\right)\right] \mathrm{d} v .
\end{aligned}
$$

According to the definition of Erdèlyi-Kober fractional integral operator (29), we have 


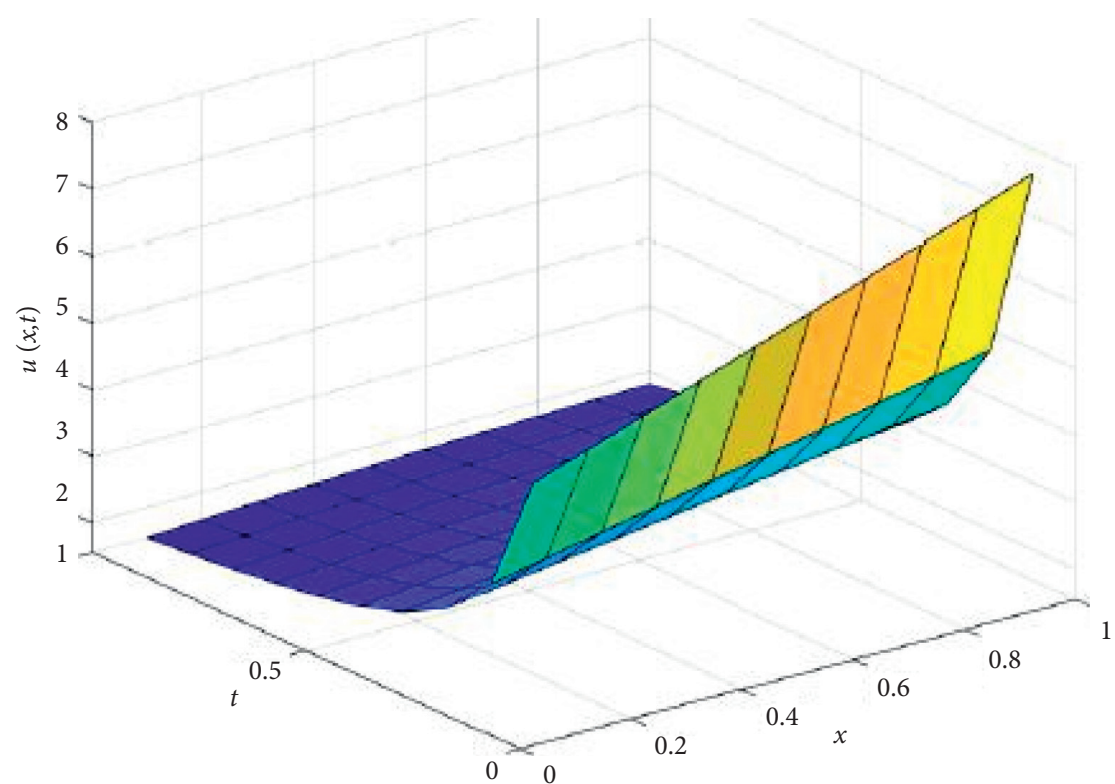

Figure 2: $3 \mathrm{D}$ plot of equation (52) with the parameter values $c_{0}=c_{1}=1, \alpha=0.75, a=2$, and $b=1$.

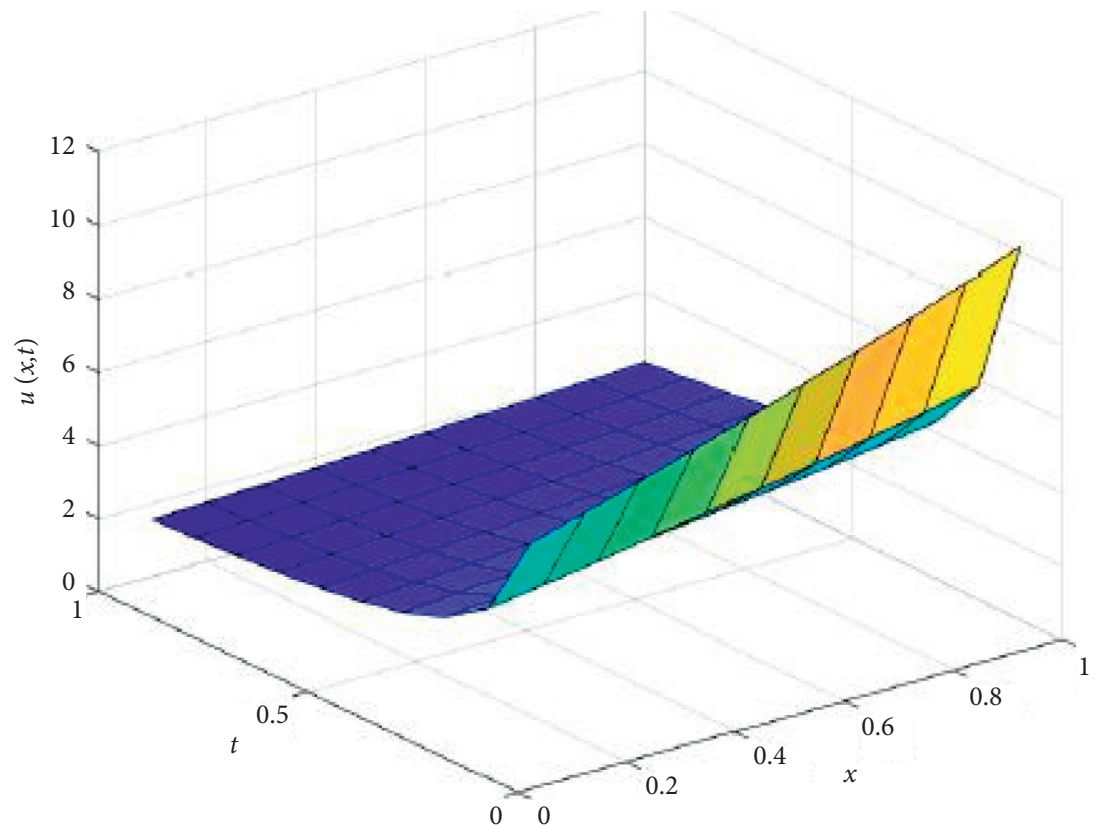

FIGURE 3: 3D plot of equation (52) with the parameter values $c_{0}=c_{1}=1, \alpha=0.90, a=2$, and $b=1$. 


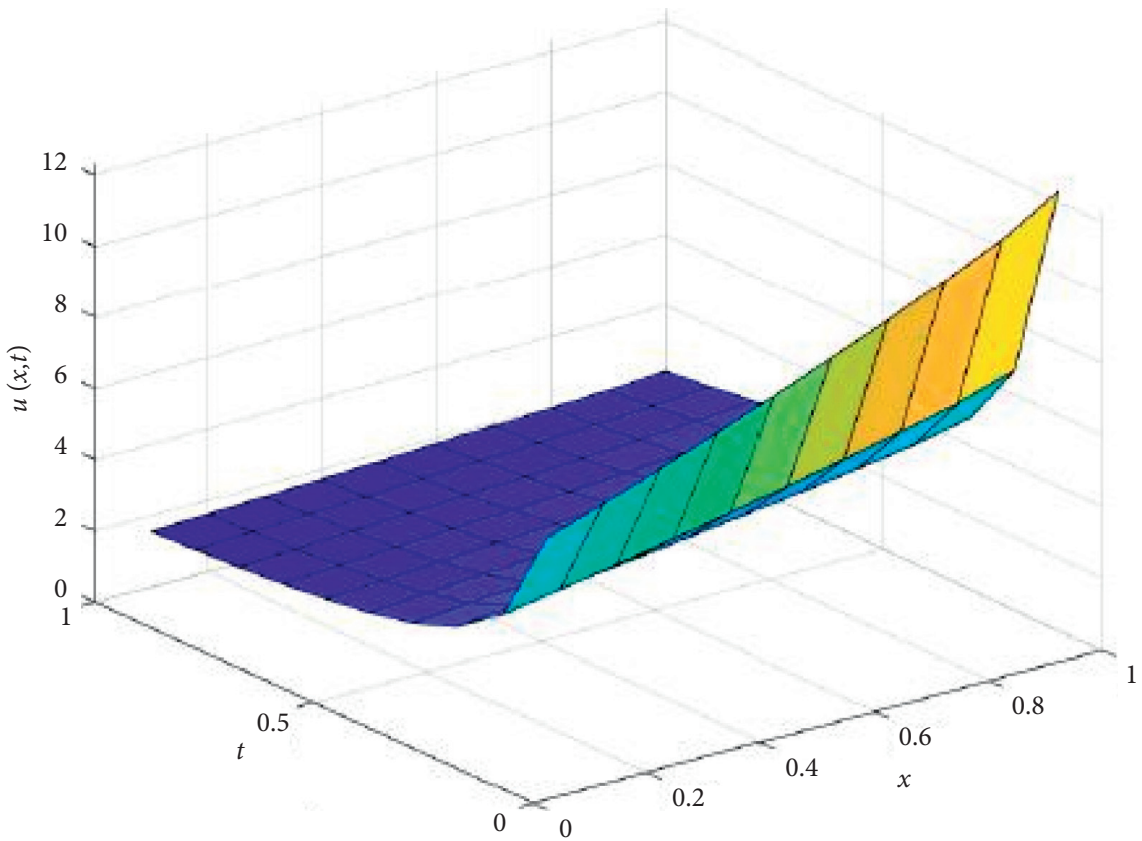

Figure 4: $3 \mathrm{D}$ plot of equation (52) with the parameter values $c_{0}=c_{1}=1, \alpha=0.99, a=2$, and $b=1$.

$$
\begin{aligned}
\frac{\partial^{n} h(z)}{\partial t^{n}} & =\frac{1}{a} \frac{\partial^{n}}{\partial t^{n}}\left[t^{n-((3 \alpha+1) / 2)}\left(K_{(2 / 1-\alpha)}^{((1-\alpha / 2), n-\alpha)} f\right)(z)\right] \\
& =\frac{1}{a} \frac{\partial^{n-1}}{\partial t^{n-1}}\left[\frac{\partial}{\partial t}\left(t^{n-((3 \alpha+1) / 2)}\left(K_{(2 / 1-\alpha)}^{((1-\alpha / 2), n-\alpha)} f\right)(z)\right)\right]
\end{aligned}
$$$$
\text { Repeating the similar procedure for } n-1 \text { times, we }
$$
obtain

$$
\begin{aligned}
\frac{\partial^{n} h(z)}{\partial t^{n}}= & \frac{1}{a} \frac{\partial^{n-1}}{\partial t^{n-1}}\left[t^{n-((3 \alpha+1) / 2)-1}\right. \\
& \left.\cdot\left(n-\frac{(3 \alpha+1)}{2}-\frac{\alpha-1}{2} z \frac{\mathrm{d}}{\mathrm{d} z}\right)\left(K_{(2 / 1-\alpha)}^{(1-\alpha / 2)+1, n-\alpha} f\right)(z)\right], \\
\vdots & \\
= & \frac{1}{a} t^{((-3 \alpha+1) / 2)} \prod_{j=0}^{n-1}\left(j-\frac{(3 \alpha+1)}{2}+1-\frac{\alpha-1}{2} z \frac{\mathrm{d}}{\mathrm{d} z}\right)\left(K_{(2 / 1-\alpha)}^{(1-\alpha / 2)+1, n-\alpha} f\right)(z) .
\end{aligned}
$$

By using equation (29), we have

$$
D_{t}^{\alpha} h(z)=\frac{1}{a} t^{-3 \alpha-1 / 2}\left(P_{(2 / \alpha-1)}^{(1-3 \alpha / 2), \alpha} f\right)(z) .
$$

According to equation (40), we have

$$
D_{t}^{\alpha} u(x, t)=D_{t}^{\alpha} h(z)+D_{t}^{\alpha}\left(\frac{2}{a}\right)
$$$$
=\frac{1}{a} t^{-3 \alpha-1 / 2}\left(P_{(2 / \alpha-1)}^{(1-3 \alpha / 2), \alpha} f\right)(z)+\frac{2}{a} \frac{t^{-\alpha}}{\Gamma(1-\alpha)} \text {. }
$$ 
Consequently, time-fractional regularized long-wave equation (1) reduces into an ordinary differential equation for fractional order with new independent variable

$$
\left(P_{(2 / \alpha-1)}^{(1-3 \alpha / 2), \alpha} f\right)(z)+2 \frac{t^{(\alpha+1 / 2)}}{\Gamma(1-\alpha)}-\frac{1}{2} f f_{z}+b \frac{(3 \alpha-1)}{2} f_{z z}=0 .
$$

The proof of the theorem is complete.

\section{Explicit Power Series Solution of Time- Fractional Regularized Long-Wave Equation}

In this section, we investigate the exact analytic solutions of equation (1) via the power series method [39]. Let us assume that

$$
f(\xi)=\sum_{n=0}^{\infty} c_{n} \xi^{n}
$$

Substituting (45) and (46) into (44), it yields

$$
\begin{aligned}
& \left(\sum_{n=0}^{\infty}\left(\frac{\Gamma((3-\alpha / 2)+n(\alpha-1) / 2)}{\Gamma((3(1-\alpha) / 2)+(n(n-1) / 2))} c_{n} \xi^{n}\right)-\frac{1}{2}\left(\sum_{n=0}^{\infty} c_{n} \xi^{n}\right)\left(\sum_{n=0}^{\infty}(n+1) c_{n+1} \xi^{n}\right)+\frac{b(3 \alpha-1)}{2} \sum_{n=0}^{\infty}(n+1)(n+2) c_{n+2} \xi^{n}\right. \\
& \quad+2 \frac{t^{((\alpha+1) / 2)}}{(\Gamma(1-\alpha))}=0 .
\end{aligned}
$$

Thus,

$$
\begin{aligned}
& \left(\sum_{n=0}^{\infty}\left(\frac{\Gamma(3-\alpha / 3+n(\alpha-1) / 2)}{\Gamma(3(1-\alpha) / 2+n(n-1) / 2)} c_{n} \xi^{n}\right)-\frac{1}{2} \sum_{n=0}^{\infty} \sum_{k=0}^{n}(n-k+1) c_{k} c_{n-k+1} \xi^{n}+\frac{b(3 \alpha-1)}{2} \sum_{n=0}^{\infty}(n+1)(n+2) c_{n+2} \xi^{n}\right. \\
& \quad+2 \frac{t^{((\alpha+1) / 2)}}{(\Gamma(1-\alpha))}=0 .
\end{aligned}
$$

Comparing coefficients in equation (47), when $n=0$, we have

$$
c_{2}=\frac{1}{b(3 \alpha-1)}\left(\frac{1}{2} c_{0} c_{1}-\frac{\Gamma((3-\alpha / 2))}{\Gamma(3(1-\alpha) / 2)} c_{0}-2 \frac{t^{((\alpha+1) / 2)}}{\Gamma(1-\alpha)}\right) .
$$

When $n \geq 1$, we get

$$
\begin{aligned}
c_{n+2}= & \frac{-2}{b(3 \alpha-1)(n+1)(n+2)} \\
& \cdot \frac{(\Gamma((3-\alpha) / 2+(n(\alpha-1)) / 2))}{(2+(n(\alpha-1)) / 2)} c_{n} \\
& \left.-\frac{1}{2} \sum_{k=0}^{n}(n-k+1) c_{k} c_{n-k+1}+2 \frac{t^{((\alpha+1) / 2)}}{\Gamma(1-\alpha)}\right) .
\end{aligned}
$$

Then, explicit solution for equation (44) can be written in the form:

$$
\begin{aligned}
f(\xi)= & c_{0}+c_{1} \xi+\frac{1}{b(3 \alpha-1)}\left(\frac{1}{2} c_{0} c_{1}-\frac{\Gamma((3-\alpha) / 2)}{\Gamma(3(1-\alpha) / 2)} c_{0}\right. \\
& \left.-2 \frac{t^{((\alpha+1) / 2)}}{\Gamma(1-\alpha)}\right) \xi^{2}-\frac{2}{b(3 \alpha-1)} \sum_{n=1}^{\infty} \frac{1}{(n+1)(n+2)} \\
& \times \frac{(\Gamma((3-\alpha) / 2)+(n(\alpha-1) / 2))}{\Gamma((3(1-\alpha) / 2)+(n(\alpha-1) / 2))} c_{n} \\
& \left.-\frac{1}{2} \sum_{k=0}^{n}(n-k+1) c_{k} c_{n-k+1}+2 \frac{t^{((\alpha+1) / 2)}}{\Gamma(1-\alpha)}\right) \xi^{n+2} .
\end{aligned}
$$


Consequently, the exact power series solution for equation (44) has the following form:

$$
\begin{aligned}
u(x, t)= & \frac{1}{a} t^{(-(\alpha+1) / 2)} c_{0}+\frac{1}{a} x t^{-\alpha} c_{1}+\frac{1}{a b(3 \alpha-1)}\left[\frac{1}{2} c_{0} c_{1}-\frac{\Gamma((3-\alpha) / 2)}{\Gamma(3(1-\alpha) / 2)} c_{0}-2 \frac{t^{((\alpha+1) / 2)}}{(\Gamma(1-\alpha))}\right] x^{2} t^{(1-3 \alpha / 2)}- \\
& \frac{2}{a b(3 \alpha-1)} \sum_{n=1}^{\infty} \frac{1}{(n+1)(n+2)}\left[\frac{\Gamma((3-\alpha / 2)+(n(\alpha-1) / 2))}{\Gamma((3(1-\alpha) / 2)+(n(\alpha-1) / 2))} c_{n}-\frac{1}{2} \sum_{k=0}^{n}(n-k+1) c_{k} c_{n-k+1}\right. \\
& \left.+2 \frac{t^{((\alpha+1) / 2)}}{(\Gamma(1-\alpha))}\right] t^{((n(1-\alpha)-3 \alpha+1) / 2)} x^{n+2} .
\end{aligned}
$$

\section{Physical Interpretation of the Power Series Solution for Equation (52)}

In order to have a clear vision of the physical properties of the power series solution and to help us analyse it, the 3dimensional plots for solution equation (52) are plotted in Figures 1-4 by using suitable parameter values.

\section{Conclusion}

In this paper, the Lie symmetry method has been successfully applied to the time-fractional regularized long-wave equation with the Riemann-Liouville fractional derivative. Generally, to find Lie point symmetries for FDEs is not an obvious task but still an interesting and efficient tool. In our context, we can obtain Lie point symmetries of the timefractional regularized long-wave equation basing on the systematic method presented by Gazizov et al. [20, 21]. The obtained nontrivial Lie point symmetries have been used to derive similarity reductions and to transform the initial equation into a nonlinear fractional ordinary differential equation with the well-known Erdèlyi-Kober fractional derivative. With the help of the power series method, the exact power series solution of the reduced FODE has been constructed. Some interesting 3-D figures for the obtained solutions were presented. Furthermore, the exact solutions obtained in this paper might be of great importance in the fields of physics and different other branches of physical sciences present the ability of our technique appear to be applicable on many various forms of nonlinear partial differential equations. There are some possible extensions of this study, e.g., symmetry analysis and conservation laws of time-space fractional (RLW) equation, which are in progress and will be discussed in the future work.

\section{Data Availability}

No data were used to support this study.

\section{Conflicts of Interest}

The authors declare that they have no conflicts of interest.

\section{References}

[1] R. L. Bagley and P. J. Torvik, "A theoretical basis for the application of fractional calculus to viscoelasticity," Journal of Rheology, vol. 27, no. 3, pp. 201-210, 1983.

[2] L. Debnath, "Recent applications of fractional calculus to science and engineering," International Journal of Mathematics and Mathematical Sciences, vol. 2003, no. 54, pp. 3413-3442, 2003.

[3] R. Hilfer, Fractional Time Evolution, Applications of Fractional Calculus in Physics, vol. 35, no. 12, pp. 87-130, Singapore, 2000.

[4] A. A. Kilbas, H. M. Srivastava, and J. J. Trujillo, Theory and Applications of Fractional Differential Equations, Vol. 204, Elsevier, Amsterdam, Netherlands, 2006.

[5] K. S. Miller and B. Ross, An Introduction to the Fractional Calculus and Fractional Differential Equations, John Wiley \& Sons, New York, NY, USA, 1993.

[6] M. Bouaouid, M. Atraoui, K. Hilal, and S. Melliani, "Fractional differential equations with non-local delay condition," Journal of Advanced Mathematical Studies, vol. 11, pp. 214-225, 2018.

[7] M. Bouaouid, K. Hilal, and S. Melliani, "Existence of mild solutions for conformable-fractional differential equations with non-local conditions," Rocky Mountain Journal of Mathematics, vol. 2019, 2019.

[8] K. Hilal and A. Kajouni, "Boundary value problems for hybrid differential equations with fractional order," Advances in Difference Equations, vol. 183, pp. 1-19, 2015.

[9] S. Kumar, A. Kumar, Z. Odibat, M. Aldhaifallah, and K. Sooppy Nisar, "A comparison study of two modified analytical approach for the solution of nonlinear fractional shallow water equations in fluid flow," AIMS Mathematics, vol. 5, no. 4, pp. 3035-3055, 2020.

[10] S. Kumar, R. Kumar, R. P. Agarwal, and B. Samet, "A study of fractional Lotka-Volterra population model using Haar wavelet and Adams-Bashforth-Moulton methods," Mathematical Methods in the Applied Sciences, vol. 43, no. 8, pp. 1-15, 2018.

[11] V. S. Kiryakova, Generalised Fractional Calculus and Applications, CRC Press, Boca Raton, FL, USA, 1993.

[12] G.-C. Wu, "A fractional variational iteration method for solving fractional nonlinear differential equations," Computers \& Mathematics with Applications, vol. 61, no. 8, pp. 2186-2190, 2011.

[13] A. Arikoglu and I. Ozkol, "Solution of fractional integrodifferential equations by using fractional differential transform method," Chaos, Solitons \& Fractals, vol. 40, no. 2, pp. 521-529, 2009. 
[14] M. M. Meerschaert, H.-P. Scheffler, and C. Tadjeran, "Finite difference methods for two-dimensional fractional dispersion equation," Journal of Computational Physics, vol. 211, no. 1, pp. 249-261, 2006.

[15] E. Aksoy, M. Kaplan, and A. Bekir, "Exponential rational function method for space-time fractional differential equations," Waves in Random and Complex Media, vol. 26, no. 2, pp. 142-151, 2016.

[16] S. Momani and Z. Odibat, "Homotopy perturbation method for nonlinear partial differential equations of fractional order," Physics Letters A, vol. 365, no. 5-6, pp. 345-350, 2007.

[17] S. S. Ray, "Analytical solution for the space fractional diffusion equation by two-step Adomian decomposition method," Communications in Nonlinear Science and Numerical Simulation, vol. 14, no. 4, pp. 1295-1306, 2009.

[18] M. Eslami, B. Fathi Vajargah, M. Mirzazadeh, and A. Biswas, "Application of first integral method to fractional partial differential equations," Indian Journal of Physics, vol. 88, no. 2, pp. 177-184, 2014.

[19] M. Bouaouid, K. Hilal, and S. Melliani, "Nonlocal telegraph equation in frame of the conformable time-fractional derivative," Advances in Mathematical Physics, vol. 2019, 2019.

[20] R. K. Gazizov, A. A. Kasatkin, and S. Yu. Lukashchuk, "Continuous transformations groups of fractional differential equations," Vestnik, USATU, vol. 9, pp. 125-135, 2007.

[21] R. K. Gazizov, A. A. Kasatkin, and S. Yu. Lukashchuk, "Symmetry properties of fractional diffusion equations," Physica Scripta, vol. T136, pp. 014-016, 2009.

[22] G. W. Wang and T. Z. Xu, "Symmetry properties and explicit solutions of the nonlinear time fractional KdV equation," Boundary value problem, vol. 232, 2013.

[23] G.-W. Wang, X.-Q. Liu, and Y.-Y. Zhang, "Lie symmetry analysis to the time fractional generalized fifth-order KdV equation," Communications in Nonlinear Science and $\mathrm{Nu}$ merical Simulation, vol. 18, no. 9, pp. 2321-2326, 2013.

[24] A. Yusuf, "Symmetry analysis, invariant subspace and conservation laws of the equation for fluid flow in porous media," International Journal of Geometric Methods in Modern Physics, vol. 17, no. 12, Article ID 2050173, 2020.

[25] R. Sahadevan and T. Bakkyaraj, "Invariant analysis of time fractional generalized Burgers and Korteweg-de Vries equations," Journal of Mathematical Analysis and Applications, vol. 393, no. 2, pp. 341-347, 2012.

[26] Q. Huang and R. Zhdanov, "Symmetries and exact solutions of the time fractional Harry-Dym equation with RiemannLiouville derivative," Physica A: Statistical Mechanics and Its Applications, vol. 409, pp. 110-118, 2014.

[27] D. Baleanu, M. Inc, A. Yusuf, and A. I. Aliyu, "Lie symmetry analysis, exact solutions and conservation laws for the time fractional Caudrey-Dodd-Gibbon-Sawada-Kotera equation," Communications in Nonlinear Science and Numerical Simulation, vol. 59, pp. 222-234, 2018.

[28] G. Bluman and S. Kumei, "Symmetries and differential equations," Springer Science and Business Media, vol. 81, 2013.

[29] P. J. Olver, Application of Lie Groups to Differential Equation, Springer, New York, NY, USA, 1986.

[30] F. Tchier and A. Yusuf, "Symmetry analysis, exact solutions and numerical approximations for the space-time Carleman equation in nonlinear dynamical systems," The European Physical Journal Plus, vol. 134, no. 6, p. 250, 2019.

[31] A. Yusuf, A. I. Aliyu, and D. Baleanu, "Conservation laws, soliton-like and stability analysis for the time fractional dispersive long-wave equation," Advances in Difference Equations, vol. 2018, no. 1, p. 319, 2018.
[32] A. Yusuf, A. I. Aliyu, and D. Baleanu, "Lie symmetry analysis and explicit solutions for the time fractional generalized Burgers-Huxley equation," Optical and Quantum Electronics, vol. 50, no. 2, p. 94, 2018.

[33] D. H. Peregrine, "Calculations of the development of an undular bore," Journal of Fluid Mechanics, vol. 25, no. 2, pp. 321-330, 1966.

[34] T. Achouri and K. Omrani, "Numerical solutions for the damped generalized regularized long-wave equation with a variable coefficient by Adomian decomposition method," Communications in Nonlinear Science and Numerical Simulation, vol. 14, no. 5, pp. 2025-2033, 2009.

[35] T. S. El-Danaf, M. A. Ramadan, and F. E. I. Abd Alaal, “The use of adomian decomposition method for solving the regularized long-wave equation," Chaos, Solitons and Fractals, vol. 26, no. 3, pp. 747-757, 2005.

[36] M. Mohammadi and R. Mokhtari, "Solving the generalized regularized long wave equation on the basis of a reproducing kernel space," Journal of Computational and Applied Mathematics, vol. 235, no. 14, pp. 4003-4014, 2011.

[37] H. Aminikhah, A. H. Refahi Sheikhani, and H. Rezazadeh, "Sub-equation method for the fractional regularized longwave equations with conformable fractional derivatives," Scientia Iranica, vol. 23, no. 3, pp. 1048-1054, 2016.

[38] D. Kumar, J. Singh, D. Baleanu, and Sushila, "Analysis of regularized long-wave equation associated with a new fractional operator with Mittag-Leffler type kernel," Physica A: Statistical Mechanics and Its Applications, vol. 492, pp. 155167, 2018.

[39] M. Inc, A. Yusuf, A. Isa Aliyu, and D. Baleanu, "Time-fractional Cahn-Allen and time-fractional Klein-Gordon equations: lie symmetry analysis, explicit solutions and convergence analysis," Physica A: Statistical Mechanics and Its Applications, vol. 493, pp. 94-106, 2018. 